\title{
Uso de indicadores para determinar la sostenibilidad de tres proyectos productivos de universidades en Costa Rica
}

\author{
Julio Arias-Reverón ${ }^{1}$, Carlos Calvo² ${ }^{2}$ Nancy Chaves ${ }^{3}$, Ma. Del Milagro Granados ${ }^{4}$, Joel Rojas Hernández ${ }^{5}$, Lorena \\ Uribe-Lorío $^{6}$ y Rodolfo WingChing-Jones ${ }^{7}$ \\ 1Escuela de Agronomía. Centro de Investigación en Protección de Cultivos.Universidad de Costa Rica. Apartado 2060. Costa Rica; \\ julio.arias@ucr.ac.cr \\ ${ }^{2}$ Sistema Nacional de Areas de Conservacion, MINAET. Costa Rica; carcalvo@costarricense.cr \\ ${ }^{3}$ Bioversity International. Centro Agronómico Tropical de Investigación y Enseñanza. Apartado 30501.Turrialba, Costa Rica; n.chaves@cgiar.org \\ ${ }^{4}$ Escuela de Agronomía. Centro de Investigación en Protección de Cultivos,. Universidad de Costa Rica. Costa Rica. Apartado 2060; \\ maria.granadosmontero@ucr.ac.cr \\ ${ }^{5}$ Escuela Internacional de Agricultura y Ganadería. Rivas, Nicaragua. Apartado 5; joelrojas3@yahoo.com \\ ${ }^{6}$ Centro de Investigación en Biología Celular y Molecular. Universidad de Costa Rica, Apartado 2060. Costa Rica; lorena.uribe@ucr.ac.cr \\ ${ }^{7}$ Escuela de Zootecnia. Centro de Investigación en Nutrición Animal-Módulo Lechero-SDA. Universidad de Costa Rica, Apartado 2060. Costa Rica; \\ rodolfo.wingching@ucr.ac.cr
}

Recibido 20-IV-2012 Corregido 24-VII-2012 Aceptado 7-VIII-2012

\begin{abstract}
Use of indicators to determine sustainability in three university production projects in Costa Rica. We implemented a matrix of indicators to estimate and compare the level of sustainability of three milk production projects in university facilities in San Carlos, Guácimo and Turrialba, Costa Rica. The method had several stages: determination of current and reference values for indicators, standardization, weighting, aggregation and graphing. We generated an aggregated index of sustainability that evaluated the development of herds in four dimensions: technical, ecological, economic and social development. The extreme index values were 0 (minimum) and 1 (optimal). The average relative yield was higher in the technical dimension $(0,36)$, followed by the economic $(0,25)$, ecological $(0,24)$ and social $(0,15)$ dimensions (herds varied). The PP-UCR presented a more balanced performance among dimensions: it presented the best performance in the technical dimension $(0,22)$, followed by the economic dimension $(0,19)$, ecological $(0,16)$ and social $(0,12)$. The PP-EARTH presented the lowest value, 0,11 in the ecological dimension, followed by social 0,10 , technical 0,08 and economic 0,01. The top project was PP-UCR with an aggregated sustainability index of 0,70 , followed by the PP-ITCR with 0,58 . The methodology allowed identification of the areas that need further attention from the point of view of sustainable development.
\end{abstract}

\section{KEY WORDS}

Livestock, indicators, sustainability, production systems, dairy, tropics, universities.

\section{RESUMEN}

Se implementó una matriz de indicadores para estimar y comparar el nivel de sostenibilidad de tres proyectos productivos dedicados a la producción láctea en instituciones de enseñanza superior, localizados en las zonas de San Carlos, Guácimo y Turrialba; Costa Rica. La metodología empleada comprendió cinco etapas: determinación de valores actuales y de referencia para los indicadores, estandarización, ponderación, agregación y graficación. A través de este método se generó un índice agregado de sostenibilidad que evaluó el desarrollo integral de las ganaderías en cuatro dimensiones: técnica, ecológica, económica y social. Los valores extremos de este índice fueron 0 (mínimo) y 1 (óptimo). Los rendimientos relativos promedio de las ganaderías en las distintas dimensiones fueron mayores en la dimensión técnica $(0,36)$, seguida por la económica $(0,25)$, ecológica $(0,24)$ y social $(0,15)$; pero se observó variación entre las ganaderías. El PP-UCR presentó el rendimiento más equilibrado entre las distintas dimensiones, ya que presentó el mejor valor en la dimensión técnica $(0,22)$, el segundo mejor rendimiento en la dimensiones económica $(0,19)$, el tercero en la ecológica $(0,16)$ y por último la dimensión social $(0,12)$. El PP-EARTH presentó los índices más bajos, 0,11 en la dimensión ecológica, 0,10 en la social, 0,08 en la técnica y 0,01 en la económica. Por último, el proyecto productivo que presentó mayor índice agregado de sostenibilidad fue la PP-UCR, con 0,70; seguida por el PP-ITCR con 0,58. La metodología permitió identificar las áreas que requieren de mayor atención en las ganaderías desde el punto de vista de desarrollo sostenible.

\section{PALABRAS CLAVE}

Ganadería, indicadores, sostenibilidad, sistemas de producción, lechería, trópico, universidades. 
La actividad ganadera en América Central ocupa alrededor del 30\% del territorio (FAO 2009) lo que la convierte en uno de los principales usos de la tierra en la región. En los últimos años, la ganadería se ha movilizado de tierras de mayor fertilidad a zonas marginales, lo cual implica ampliar las áreas ganaderas para satisfacer la creciente demanda de productos de origen animal. Esto conlleva a un mayor riesgo en cuanto a degradación de los recursos naturales tales como suelos, bosques, agua y biodiversidad (Villanueva et al. 2008). Sin embargo, es innegable la importancia socioeconómica que representa el sector ganadero para la región, por ello es importante el diseño y rediseño de modelos productivos encaminados a la mejora de los medios de vida de todos los involucrados en las diferentes cadenas de producción, tanto de leche como de carne, y que además sean responsables con el ambiente (Villanueva et al. 2008).

En el ámbito de las instituciones de educación superior, se concibió un modelo físico o prototipo de un sistema de producción de ganadería de leche, denominado Módulo Lechero, con posibilidad de adaptarse a la situación social y económica de los productores de pequeña escala (Pezo et al. 1993). Además de requerir una pequeña extensión de tierra, estos módulos maximizan la utilización de la mano de obra, procuran la disminución de costos de producción, el aumento de ingresos y la reducción de impactos ambientales negativos, para servir como modelos productivos sostenibles (Pezo et al. 1993).

Desde el punto de vista de sostenibilidad, el sistema ganadero debe garantizar condiciones socioeconómicas adecuadas para el grupo familiar, así como la obtención de productos de origen animal de buena calidad sin comprometer en el proceso la base de recursos naturales presente en el sistema mismo (Ruiz \& Oregui 2001). Para estimar la sostenibilidad se utilizan indicadores, que son herramientas para resumir y simplificar, de manera útil, información de naturaleza compleja. Pueden ser números o cualidades que evidencian el estado o condición de un proceso o fenómeno en relación con la sostenibilidad y permiten entender cómo evolucionan las cosas a través del tiempo (De Camino y Müller 1993, Winograd 1995, Müller 1996). No obstante se han utilizado en numerosos estudios para determinar la sostenibilidad de diversos sistemas productivos, es preciso tomar en cuenta que los indicadores no son universales, sino más bien que deben ser ajustados a las necesidades de información según los objetivos de cada estudio para la toma de determinadas decisiones (Murillo et al. 2004).

En un estudio en Costa Rica, Murillo et al. (2004) informan de rendimientos relativos promedio en la dimensión social $(0,78)$, económica $(0,58)$, ecológica $(0,56)$ y técnica
$(0,49)$, en cinco fincas ganaderas, pero también describen que entre los datos se observó una alta variación entre las ganaderías. Por lo que el objetivo del presente estudio fue el de comparar el nivel de sostenibilidad ambiental, técnica, económica y social de tres proyectos productivos de instituciones de enseñanza superior, localizados en San Carlos, Guácimo y Turrialba, Costa Rica.

\section{METODOLOGÍA}

Este estudio se llevó a cabo durante el año 2010 en tres proyectos productivos (PP) cuyo objetivo principal es la producción láctea. Al ser PP de centros de enseñanza superior, cumplen funciones de docencia, acción social e investigación. Estos centros se ubican en la Universidad de Costa Rica Sede del Atlántico, Recinto Turrialba, el Instituto Tecnológico de Costa Rica, en su Sede de Santa Clara y la Universidad EARTH en Guácimo. Estos sistemas fueron seleccionados tomando en consideración la disponibilidad de información en cuanto a registros productivos, reproductivos, sanitarios y nutricionales, así como la anuencia del administrador para facilitar la información complementaria (análisis de suelos, pastos, salarios, precios de venta, entre otros).

Para tal fin se siguió la metodología propuesta por Murillo et al. 2004 (a y b) para la estimación de un índice agregado de sostenibilidad en cada una de las fincas estudiadas, no incluyendo los índices para los cuales no exista información en dichas fincas. En este sentido se trabajó con los índices descritos en el Cuadro 1, para las dimensiones económica, ecológica, técnica y social.

\section{Sistema de referencia}

Se desarrolló un sistema de referencia de valores óptimos y subóptimos que permitieran comparar de manera desagregada los sistemas de producción en el tiempo y espacio, es decir, los valores obtenidos en cada finca en comparación con los valores de referencia (Müller 1996; Hünnemeyer et al. 1997). En este estudio se buscó la manera más sencilla para determinar el óptimo y el subóptimo, tomando como referencia los valores más altos o más bajos encontrados en los tres proyectos productivos, por ejemplo:

- Para el indicador $\mathrm{Kg}$ de leche anual por $\mathrm{m}^{2}$ de construcción, el valor más alto en las tres fincas fue de 133, siendo este el valor óptimo, mientras que, el valor más bajo en las tres fincas fue de 30,6, considerado el valor subóptimo (Fig. 1). 


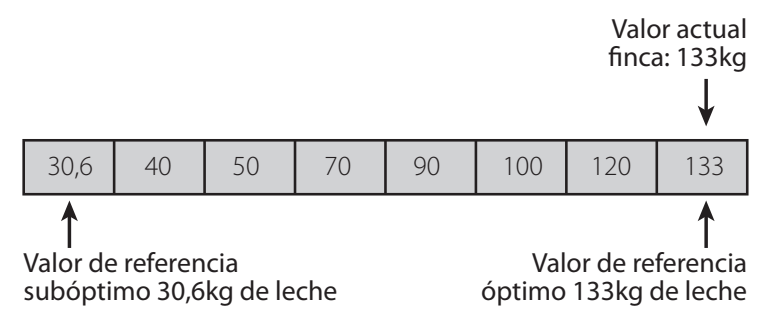

FIG. 1. Sistema de referencia elaborado para determinar valores óptimos y subóptimos a partir de los indicadores obtenidos en los tres PP de centros de enseñanza superior localizados en Turrialba, Santa Clara y Guácimo, Costa Rica.

\section{Estandarización}

Los valores obtenidos para cada indicador se convertiron a valores estándares, con un valor óptimo de 1 y un subóptimo de 0 (Fig. 2). Para ello se aplicó la siguiente fórmula (Murillo et al. 2004b): donde, RR = Rendimiento relativo, $\mathrm{Va}=$ Valor actual, Vso $=$ Valor subóptimo y Vo = Valor óptimo.

$$
R R=\frac{V_{a}-V_{s o}}{V_{o}-V_{s o}} \quad[1]
$$

Tomando el dato anterior y aplicando esta fórmula, el resultado es el siguiente: el rendimiento relativo para un $\mathrm{PP}$ en producción de $\mathrm{kg}$ de leche/ $\mathrm{m}^{2}$ es de 1 (Fig. 2). Es decir, $\mathrm{RR}=(133-30,6 / 133-30,6)=1$

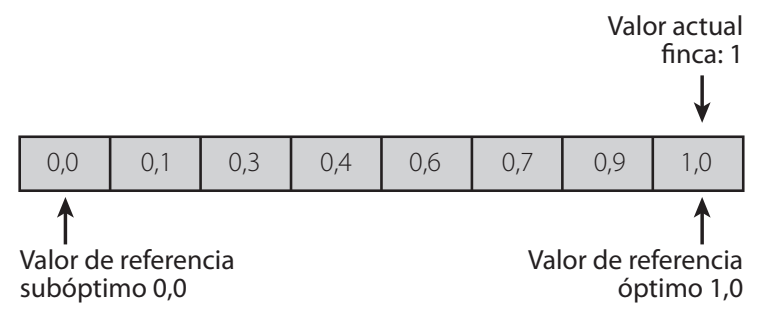

FIG. 2. Estandarización de los datos elaborado para determinar valores óptimos y subóptimos a partir de los indicadores obtenidos en los tres PP de centros de enseñanza superior localizados en Turrialba, Santa Clara y Guácimo, Costa Rica.

\section{Ponderación}

Se realizó mediante el método de ranqueo completo (Looijen 1997; Murillo et al. 2004a; González 2005). El panel de evaluadores estuvo conformado por un ecólogo, dos zootecnistas, tres agrónomos y un forestal. La fórmula que se utilizó fue la descrita por Murillo et al. ( 2004b), donde $\mathrm{FPi}=$ Valor ponderado dado a cada indicador $(0<$ $\mathrm{FPi} \leq 1), \mathrm{Se}=$ Score (calificación) dado al indicador i: según evaluador e $(1 \leq \mathrm{Se} \leq \mathrm{ni})$, ni = número de indicadores y ne $=$ número de evaluadores.

$$
F P i=\frac{\sum_{e=1}^{n e} S e}{\sum_{i=1}^{n i} \sum_{e=1}^{n e} S e}
$$

Está misma fórmula se utilizó para obtener el factor de ponderación por componente (FPc) (suelo-agua, plantasalimentos, humano, infraestructura y animal) y dimensión (FPd) (ecológica, económica, social y técnica).

\section{Índice agregado de sostenibilidad por componente}

Se determinó mediante la sumatoria del rendimiento relativo por la ponderación de cada indicador, para ello se aplicó la siguiente fórmula (Murillo et al. 2004b): donde IASC = Índice de sostenibilidad agregado por componente, $\mathrm{RRi}=$ Rendimiento relativo de cada indicador y $\mathrm{FPi}=$ Factor ponderado dado a cada indicador.

$$
I A S_{c}=\sum_{i=1}^{n}\left(R R_{i} \times F P_{i}\right)
$$

\section{Índice agregado de sostenibilidad por dimensión}

Se determinó mediante la sumatoria de los productos del índice agregado de cada componente por su factor de ponderación, se aplicó la siguiente fórmula (Murillo et al. 2004b): donde IASd = Índice agregado de sostenibilidad por dimensión, IASC = Índice agregado de sostenibilidad por componente y FPc = Factor ponderado dado a cada componente.

$$
I A S_{d}=\sum_{c=1}^{n}\left(I A S_{c} \times F P_{c}\right)
$$


CUADRO 1

Indicadores de sostenibilidad de los componentes dentro de la dimensión económica, ecológica, técnica y social evaluados en los tres proyectos productivos (PP) de centros de enseñanza superior localizados en Turrialba, Santa Clara y Guácimo, Costa Rica.

\begin{tabular}{|c|c|c|}
\hline Dimensiones & Componentes & Indicadores * \\
\hline \multirow{4}{*}{ 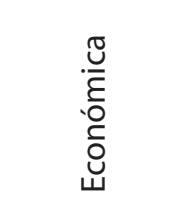 } & Infraestructura y Equipo & Ingresos anuales $/ \mathrm{m}^{2}$ de construcción \\
\hline & Animales & Ingreso neto/animal o UA \\
\hline & Suelos-Agua & Ingreso total año/ha de potreros \\
\hline & Recursos Humanos & Costo $\mathrm{MO} / \mathrm{kg}$ de leche \\
\hline \multirow{7}{*}{$\begin{array}{l}\frac{0}{\overline{0}} \\
\frac{.0}{0} \\
\text { U }\end{array}$} & Infraestructura y Equipo & $\begin{array}{l}\mathrm{L} \text { agua/m²/año; Drenaje a fuentes de agua/drenaje a los } \\
\text { potreros; Tratamiento de las aguas de lavado (Sí o no) }\end{array}$ \\
\hline & \multirow{2}{*}{ Suelos-Agua } & Fósforo disponible (ppm) en los potreros \\
\hline & & Potasio extraíble (Meq/100 g) en los potreros \\
\hline & \multirow{2}{*}{ Recursos Humanos } & Escala de valores $(1-5)$ \\
\hline & & Escala de valores $(1-5)$ \\
\hline & \multirow{2}{*}{ Plantas } & $\begin{array}{l}\mathrm{kg} \text { de MS requerida en vacas en producción/kg de MS en la } \\
\text { dieta suministrada }\end{array}$ \\
\hline & & $\begin{array}{l}\text { PC en el forraje/PC suministrada en la dieta en vacas en } \\
\text { producción }\end{array}$ \\
\hline \multirow{9}{*}{ 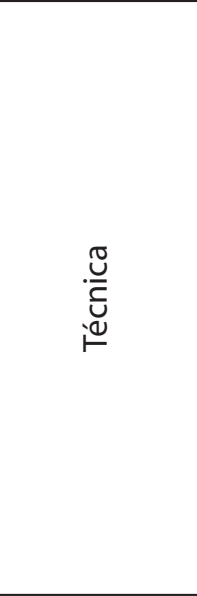 } & \multirow{2}{*}{ Infraestructura y Equipo } & $\mathrm{kg}$ de leche anual $/ \mathrm{m}^{2}$ de construcción \\
\hline & & $\mathrm{kg}$ de carne anual $/ \mathrm{m}^{2}$ de construcción \\
\hline & Animales & $\begin{array}{l}\text { kg leche/vaca/día; kg leche/vaca/lactancia; \% Vacas en ordeño; } \\
\text { Edad al primer parto (meses); } \\
\text { Intervalo entre partos (días); Servicios por concepción }\end{array}$ \\
\hline & Suelos-Agua & $\begin{array}{l}\text { Balance de N-P-K en los potreros (requerido/aplicado) } \\
\text { kg de leche año/ha de potreros }\end{array}$ \\
\hline & \multirow{2}{*}{ Recursos Humanos } & UA/Mano de obra \\
\hline & & kg de leche/Mano de obra \\
\hline & \multirow{3}{*}{ Plantas } & $\mathrm{kg}$ de MS/ha/año en los potreros \\
\hline & & kg de PC en los forrajes/ha/año \\
\hline & & Carga animal (UA/ha/ año) \\
\hline \multirow{6}{*}{ : } & \multirow{4}{*}{ Infraestructura y Equipo } & Capacitación en manejo de equipos (Sí o No) \\
\hline & & Seguridad laboral en las instalaciones y equipos \\
\hline & & Existe botiquín de primeros auxilios (Sí o No) \\
\hline & & Salario real contra Salario mínimo \\
\hline & \multirow[t]{2}{*}{ Recursos Humanos } & Seguro Social (Si o No) \\
\hline & & Presencia/Ausencia de medidas de bioseguridad \\
\hline
\end{tabular}

*MO=Mano de obra, MS= Materia seca, $\mathrm{PC}=$ Proteína cruda, $\mathrm{UA}=$ Unidades animales 


\section{Índice agregado de sostenibilidad para el sistema de producción}

Por último se determinó el índice integral de sostenibilidad del sistema (PP), mediante la sumatoria del índice agregado de cada dimensión por su factor de ponderación, utilizando la siguiente fórmula (Murillo et al. 2004b), donde IASs: Índice agregado de sostenibilidad para el sistema, IASd: Índice agregado de sostenibilidad por dimensión y VPd: Valor ponderado dado a cada dimensión.

$$
\mathrm{ISI}=\sum_{d=1}^{n}\left(I A S_{d} \times F P_{d}\right)
$$

\section{RESULTADOS}

\section{Ponderación de Componentes}

En el Cuadro 2 se muestran las diferentes calificaciones dadas por los evaluadores mediante el procedimiento de ranqueo completo. Los resultados muestran que en la dimensión económica el componente animales obtiene la mayor importancia con un valor de ponderación de 0,39, producto que los evaluadores le brindan mayor puntuación; se observa en esta dimensión que los evaluadores dan un orden de puntuación similar a cada componente. En la dimensión ecológica el componente plantas obtiene una calificación de 0,4; se aprecia en esta dimensión una variación en la puntuación realizada por los evaluadores en los componentes infraestructura y equipo, y recursos humanos. En la dimensión técnica también se puede apreciar variaciones en las calificaciones de los componentes; sin embargo el componente con mayor valor de ponderación es infraestructura y equipo con 0,25 , muy similar a animales y recursos humanos. Por último en la dimensión social, compuesta de dos componentes, sólo el evaluador 4 difiere del resto del grupo, obtiendo así la variable recursos humanos un valor de 0,62.

\section{Ponderación de Dimensiones}

En el Cuadro 3, de igual manera que en la ponderación por componentes, se observa la valoración que realiza cada uno de los evaluadores en las dimensiones estudiadas en los tres proyectos productivos. Obteniendo así variaciones en las calificaciones hechas; sin embargo el mayor valor lo obtiene la dimensión técnica con 0,36 , solo el evaluador 1 difiere del resto del grupo. En el caso del resto de las dimensiones se puede apreciar que no hay similitud de criterios en la calificación realizada por los evaluadores.

\section{Dimensión económica}

El PP-UCR supera al PP del ITCR y de la EARTH en tres de los cuadro componentes que componen esta dimensión, a saber, infraestructura y equipo, animales y suelos-agua (Cuadro 4). En cambio, el PP-ITCR obtiene el mayor valor promedio en el componente referente a recursos humanos. En el 75\% de los componentes evaluados el PP-EAR$\mathrm{TH}$, presenta los menores valores promedio obtenidos.

\section{Dimensión ecológica}

Con respecto a esta dimensión, se determina una mayor similitud entre valores promedios obtenidos en cada indicador para los PP, excepto en el componente sueloagua, donde la brecha es igual a 0,95 unidades porcentuales (Cuadro 4). En el componente infraestructura y equipo el PP-ITCR obtiene el mayor valor, mientras que, en el componente suelos-agua, esta posición es para el PP-UCR. En el caso del componente de recursos humanos, es el PPEARTH el que obtiene el valor mayor con respecto a los otros dos sistemas. Por último, en el componente planta, el PP-ITCR y PP-EARTH presenta un valor de 0,57, valor que supera al PP-UCR en 0,14 .

\section{Dimensión técnica}

En el $60 \%$ de los componentes evaluados, el PP-UCR obtiene el valor mayor, mientras que el PP-EARTH presenta el $60 \%$ de los valores bajos, fluctuando esta diferencias entre 0,5 y 0,24 (Cuadro 4). En el caso del PP-ITCR, presentó el mayor valor en el componente de recursos humanos, superando al PP-EARTH y PP-UCR en 0,46 y 0,48 unidades.

\section{Dimensión social}

Esta dimensión fue evaluada por dos componentes, en el que se considera la infraestructura y equipo, en donde los tres proyectos productivos alcanzan un valor de 1 , mientras que, en el componente de recursos humanos, el PP-UCR supera en 1,47 veces a los PP-ITCR y el PP-EARTH (Cuadro 4).

\section{Sostenibilidad de las dimensiones dentro de los proyectos productivos}

En el caso de la dimensión económica, el PP-UCR y PPITCR obtienen un valor de 0,70 y 0,69 respectivamente, valor 17,5 veces mayor al obtenido por el PP-EARTH (Fig. 3). Con respecto a la dimensión ecológica, los valores obtenidos fluctuaron entre 0,67 y 0,46 , lo que indica una sostenibilidad superior entre el valor mayor y el menor de 1,46. En este caso, el PP-UCR obtiene el valor mayor mientras que el valor menor fue para el PP-EARTH. En esta dimensión, el PP-ITCR obtuvo un valor de 0,49, valor 0,18 inferior al obtenido para el PP-UCR. En la dimensión técnica, el 
CUADRO 2

Ponderación mediante ranqueo completo para los componentes dentro de las cuatro dimensiones evaluados en los tres proyectos productivos de centros de enseñanza superior localizados en Turrialba, Santa Clara y Guácimo, Costa Rica.

\begin{tabular}{|c|c|c|c|c|c|c|c|c|c|c|}
\hline Dimensiones & Componentes & Eva_1 & Eva_2 & Eva_3 & Eva_4 & Eva_5 & Eva_6 & Eva_7 & Suma & Pond \\
\hline \multicolumn{11}{|c|}{ Método de Ranqueo Completo: "n” más importante y 1 menos importante } \\
\hline \multirow{5}{*}{ 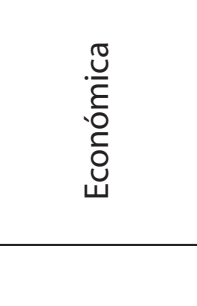 } & $\begin{array}{l}\text { Infraestructura y } \\
\text { Equipo }\end{array}$ & 1 & 1 & 1 & 1 & 1 & 1 & 1 & 7 & 0,10 \\
\hline & Animales & 4 & 4 & 4 & 3 & 4 & 4 & 4 & 27 & 0,39 \\
\hline & Suelos - Agua & 3 & 2 & 2 & 2 & 2 & 3 & 2 & 16 & 0,23 \\
\hline & Recursos Humanos & 2 & 3 & 3 & 4 & 3 & 2 & 3 & 20 & 0,29 \\
\hline & & 10 & 10 & 10 & 10 & 10 & 10 & 10 & 70 & 1,00 \\
\hline \multirow{5}{*}{ 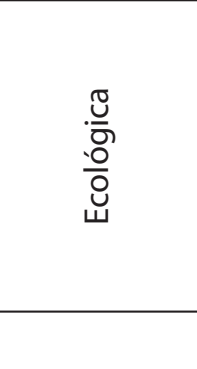 } & $\begin{array}{l}\text { Infraestructura y } \\
\text { Equipo }\end{array}$ & 1 & 1 & 1 & 2 & 2 & 1 & 1 & 9 & 0,1 \\
\hline & Suelos - Agua & 3 & 3 & 3 & 3 & 3 & 4 & 4 & 23 & 0,3 \\
\hline & Recursos Humanos & 2 & 2 & 2 & 1 & 1 & 3 & 2 & 13 & 0,2 \\
\hline & Plantas & 4 & 4 & 4 & 4 & 4 & 2 & 3 & 25 & 0,4 \\
\hline & & 10 & 10 & 10 & 10 & 10 & 10 & 10 & 70 & 1,0 \\
\hline \multirow{6}{*}{$\frac{\mathbb{U}}{\stackrel{U}{C}}$} & $\begin{array}{l}\text { Infraestructura y } \\
\text { Equipo }\end{array}$ & 5 & 5 & 3 & 5 & 3 & 4 & 1,5 & 26,5 & 0,25 \\
\hline & Animales & 3 & 4 & 2 & 4 & 4 & 3 & 5 & 25 & 0,24 \\
\hline & Suelos - Agua & 1 & 2 & 1 & 3 & 2 & 1 & 3 & 13 & 0,12 \\
\hline & Recursos Humanos & 4 & 3 & 5 & 1 & 5 & 5 & 1,5 & 24,5 & 0,23 \\
\hline & Plantas & 2 & 1 & 4 & 2 & 1 & 2 & 4 & 16 & 0,15 \\
\hline & & 15 & 15 & 15 & 15 & 15 & 15 & 15 & 105 & 1,00 \\
\hline \multirow{3}{*}{$\begin{array}{l}\bar{\pi} \\
\frac{\pi}{0} \\
\text { ㄴ. }\end{array}$} & $\begin{array}{l}\text { Infraestructura y } \\
\text { Equipo }\end{array}$ & 1 & 1 & 1 & 2 & 1 & 1 & 1 & 8 & 0,38 \\
\hline & Recursos Humanos & 2 & 2 & 2 & 1 & 2 & 2 & 2 & 13 & 0,62 \\
\hline & & 3 & 3 & 3 & 3 & 3 & 3 & 3 & 21 & 1,00 \\
\hline
\end{tabular}


CUADRO 3

Ponderación mediante ranqueo completo para las dimensiones evaluados en los tres proyectos productivos de centros de enseñanza superior localizados en Turrialba, Santa Clara y Guácimo, Costa Rica.

\begin{tabular}{lccccccccc}
\hline Dimensiones & Eva_1 & Eva_2 & Eva_3 & Eva_4 & Eva_5 & Eva_6 & Eva_7 & Suma & Ponderación \\
\hline Social & 1,5 & 1 & 1 & 3 & 1 & 1 & 2 & 10,5 & 0,15 \\
Técnica & 1,5 & 4 & 4 & 4 & 4 & 4 & 4 & 25,5 & 0,36 \\
Ecológica & 3,5 & 3 & 2 & 2 & 2 & 3 & 1 & 16,5 & 0,24 \\
Económica & 3,5 & 2 & 3 & 1 & 3 & 2 & 3 & 17,5 & 0,25 \\
& 10 & 10 & 10 & 10 & 10 & 10 & 10 & 70 & 1,00
\end{tabular}

CUADRO 4

Comparación de los índices de sostenibilidad de los componentes dentro de la dimensión económica, ecológica, técnica y social de los en los tres proyectos productivos de centros de enseñanza superior localizados en Turrialba, Santa Clara y Guácimo, Costa Rica.

\begin{tabular}{llccc}
\hline \multirow{2}{*}{ Dimensiones } & \multirow{2}{*}{ Componentes } & \multicolumn{3}{c}{ Proyecto productivo } \\
\cline { 3 - 5 } Económica & UCR & ITCR & EARTH \\
\hline & Infraestructura y Equipo & 1,00 & 0,88 & 0,00 \\
& Snimales & 1,00 & 0,68 & 0,00 \\
& Suelos-Agua & 0,93 & 0,23 & 0,01 \\
& Recursos Humanos & 0,00 & 1,00 & 0,12 \\
\multirow{5}{*}{ Ecológica } & Infraestructura y Equipo & 0,57 & 0,98 & 0,62 \\
& Suelos-Agua & 1,00 & 0,05 & 0,05 \\
& Recursos Humanos & 0,63 & 0,75 & 0,88 \\
& Plantas & 0,43 & 0,57 & 0,57 \\
Técnica & Infraestructura y Equipo & 0,75 & 0,56 & 0,33 \\
& Animales & 0,63 & 0,55 & 0,11 \\
& Suelos-Agua & 0,24 & 0,05 & 0,00 \\
\multirow{2}{*}{ Social } & Recursos Humanos & 0,36 & 0,84 & 0,38 \\
& Plantas & 1,00 & 0,05 & 0,08 \\
& Infraestructura y Equipo & 1,00 & 1,00 & 1,00 \\
& Recursos Humanos & 1,00 & 0,68 & 0,68
\end{tabular}


PP-UCR supera en 1,27 y 2,90 veces los valores obtenidos en el PP-ITCR y PP-EARTH respectivamente. Mientras que la relación entre el PP-ITCR y PP-EARTH es de 2,28 veces mayor en el proyecto productivo del Instituto Tecnológico de Costa Rica. Por último, en la dimensión social, los valores obtenidos para el PP-UCR, PP-ITCR y PP-EARTH fueron de $1,0,80$ y 0,80 respectivamente.

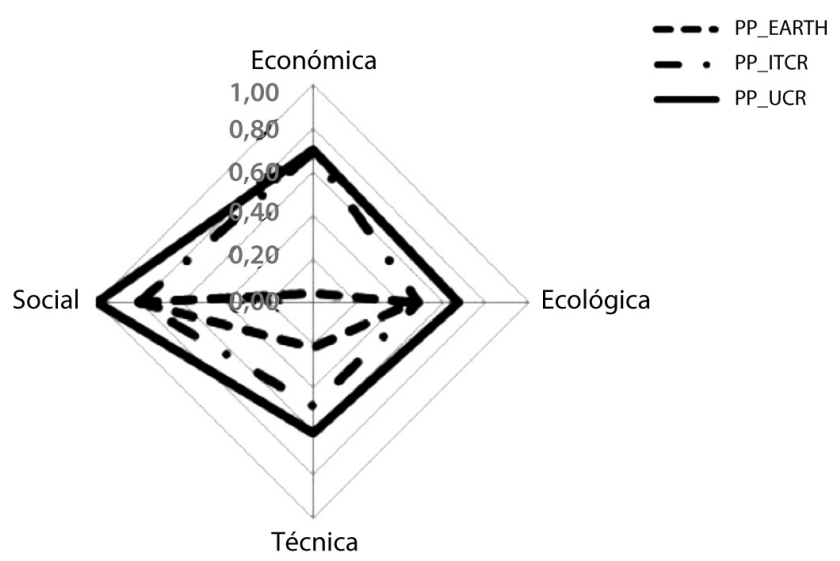

FIG. 3. Índice de sostenibilidad agregado para las dimensiones económicas, ecológica, técnica y social en los tres proyectos productivos de centros de enseñanza superior localizados en Turrialba, Santa Clara y Guácimo, Costa Rica.

\section{Índice de sostenibilidad agregado para los proyectos productivos}

Los valores obtenidos indican que el PP-UCR presentó el valor mayor con respecto a los otros dos proyectos productivos $(0,70)$. Este valor superó en 1,20 y 2,19 veces el valor obtenido para el PP-ITCR $(0,58)$ y el PP-EARTH $(0,32)$ respectivamente. En el caso de la relación entre el PP-ITCR y el PP-EARTH, favorece al primero en 1,58 veces.

\section{DISCUSIÓN}

Al utilizar un método de ponderación en el análisis de los sistemas productivos, se busca integrar el juicio de valor de los evaluadores de una manera objetiva. Es recomendable que en este proceso los expertos sean de diferentes disciplinas, socioeconómica, ecológica y de producción animal (Murillo et al. 2004b). Los resultados obtenidos en la ponderación de esta investigación, se observa que en la dimensión económica el componente animales es el que obtiene la mayor calificación $(0,39)$, seguido de recursos humanos; esto difiere a lo encontrado por Murillo et al. (2004b) donde fue el componente vegetal el que obtuvo el mayor valor, seguido del componente animal. En la dimensión ecológica el componene plantas obtiene el valor más alto $(0,4)$, seguido de suelo $(0,3)$, diferente a lo encontrado por Murillo et al. (2004b), donde suelo es el valor mayor $(0,4)$, seguido de plantas con $(0,3)$. En la dimensión técnica, el componente de mayor valor es infraestructura $(0,25)$ y le sigue animales $(0,24)$, en el caso de Murillo et al. (2004b), el componente animal es el mayor (0,31), seguido de plantas $(0,28)$. En la dimensión social, este estudio coincide con Murillo et al. (2004b), en que el componente humano es el más impornte. Es necesario mencionar que estas divergencias pueden deberse a la percepción e importancia que cada evaluador experto brinde a cada uno los componentes en la dimensión.

En la ponderación de las dimensiones se encontró que lo técnico es el de mayor importancia $(0,36)$, en segundo lugar lo económico $(0,25)$, cerca de lo ecológico $(0,24)$ y lo social en último $(0,15)$. Esto también difiere a lo encontrado por Murillo et al. (2004b), donde lo económico es lo primero $(0,32)$, seguido de lo técnico $(0,26)$, en tercer lugar está lo ecológico y lo social (0,21 respectivamente).

$\mathrm{Al}$ analizar estos tres proyectos productivos de forma integral, se notan diferencias a nivel de manejo nutricional, tipo de animal y organización del sistema. En el caso del PP-UCR, este es un sistema de producción intensivo, el cuál presenta una relación ingreso total de 5,6 millones de colones por ha de porteros. Los animales pastorean pasto Estrella africana (Cynodon nlemfuensis) a 25 días y basa su producción en el manejo de animales Jersey. En el caso del PP-ITCR, no presenta una raza definida, si no la mezcla entre ganado Jersey, Holstein y sus cruces, pastoreo de pasto Ratana (Ishaemun ciliare) y de pasto Brachiaria, y para este trabajo, se le cálculo un ingreso por ha de potreros de 1,6 millones de colones. De forma similar, el PP-EARTH, presenta el pastoreo del pasto Ratana, presenta un animal producto del cruce animales Bos taurus y Bos indicus, el cual presenta aptitud de animal doble propósito. Este sistema de producción genera un ingreso total al año por ha de potreros de 0,45 millones de colones. Por otro lado, el PP-UCR, presenta el costo de mano de obra por litro de leche más alto de los tres sistemas productivos, debido a que presenta los salarios más altos para su personal. Resultados que explican el comportamiento de los sistemas hacia la sostenibilidad en las dimensiones evaluadas (Fig. 4).

Al ser el PP-UCR un sistema intensivo, el uso de fertilizantes sintéticos para la producción de forraje reduce los indicadores de sostenibilidad del sistema, ya que presenta ingresos de insumos al sistema, lo cual aumenta su 


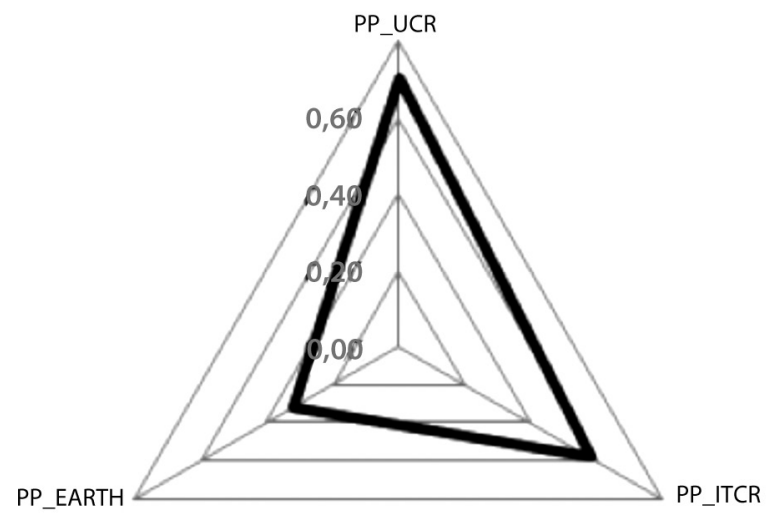

FIG. 4. Sostenibilidad aproximada de los tres proyectos productivos de centros de enseñanza superior localizados en Turrialba, Santa Clara y Guácimo, Costa Rica.

dependencia. En el caso de los alimentos balanceados de uso comercial para optimizar la producción de los animales, a nivel nutricional mejora el perfil de nutrimentos que se le aporta a los animales, lo cual se traduce en una mejora en la producción, condición corporal y la reproducción de los animales, situación que se refleja en valores inferiores de sostenibilidad en la dimensión ecológica.

A nivel tecnológico, las características de los animales presentes en cada sistema, como el manejo técnico que se les aplica permite que los animales del PP-UCR tengan una producción de leche por lactancia de 5,192 litros, mientras que en el PP-ITCR y el PP-EARTH, la producción es de 1,633 y 1,126 litro por animal. Como se comentó, la producción de leche en el PP-UCR, esta influenciada por el aporte de $\mathrm{N}$ al suelo por fuentes sintéticas para la producción de forrajes, y el incremento de la densidad de nutrimentos al uso de fuentes de alimento, insumos que mejoran la sostenibilidad del sistema en la dimensión técnica, pero la castigan en la dimensión ecológica.

Por último, al ser modelos productivos de enseñanza superior y por la legislación laboral presente en Costa Rica, los empleados de estos proyectos productivos están protegidos por la Ley de Protección al Trabajador, Ley No 7983 del 16 de febrero del 2000, por lo cual no se presentaron diferencias en la dimensión social.

En el caso del índice de sostenibilidad y la aproximación a la sostenibilidad de los sistemas, se nota que el proyectos más balanceado es el PP-UCR, comportamiento que puede ser explicado por las características de este modelo productivo, en donde hace un uso estratégico de los recursos, para aumentar la producción de forraje de buena calidad, lo que repercute en la producción de leche. Al comparar los resultados obtenidos en esta investigación con los informados por Murillo et al. (2004b), se determina que estos sistemas presentan valores similares o mayores a los estudiados por estos autores, donde se determinó un rango de 0,50 a 0,65 para este índice. Igualmente, los valores obtenidos en esta investigación, se encuentran dentro los rangos descritos en seis sistemas comerciales de producción en Costa Rica, para las dimensiones técnica $(0,35$ a 0,75$)$, social $(0,60$ a 0,85$)$, económica $(0,45$ a 0,80$)$ y ecológica $(0,45$ a 0,80) (Murillo et al. 2004b). Lo que podría demostrar, que los PP manejados por centros de enseñanza superior, dentro de su dinámica de funcionamiento, se comportan mejor que sistemas comerciales de producción. En el caso del índice de sostenibilidad, solo el PPEARTH, presentó un valor 0,10 unidades menor al rango mínimo obtenido por Murillo et al. (2004b), lo cual podría deberse a una subutilización del recurso suelo y forraje.

Los proyectos productivos de instituciones de enseñanza superior presentan características productivas diferentes, los cuales son establecidos por los intereses institucionales de cada uno. Lo que permite obtener las diferencias entre los PP-UCR, PP-ITCR y PP-EARTH. Este ejercicio acerca de la sostenibilidad de los sistemas, es una llamada de atención para los responsables de estos sistemas, para que realicen un análisis del mismo, para la toma de decisiones, con la intención de alcanzar una estabilidad económica, ecológica, técnica y social, y así, proyecten estos modelos productivos a otros productores.

\section{REFERENCIAS}

De Camino, R. \& S, Müller. 1993. Sostenibilidad de la agricultura y los recursos naturales: bases para establecer indicadores. IICA, San José, Costa Rica.

FAO (Organización de las Naciones Unidas para la Agricultura y la Alimentación, IT). 2009. Bases de datos FAOSTAT. Roma, IT. (Disponible en http://faostat.fao.org/site/; Consultado 29 de set 2011)

González, VJ. 2005. Evaluación de alternativas de desarrollo sostenible en hatos de lechería especializada y doble propósito de la Escuela Centroamericana de Ganadería. Tesis Mag. Sc. Posgrado Regional en Ciencias Veterinarias Tropicales. Universidad Nacional, Heredia, CR.

Hünnemeyer, AJ, R de, Camino \& S., Müller. 1997. Análisis del desarrollo sostenible en Centroamérica. Indicadores para la agricultura y los recursos naturales. IICA/GTZ. San José. Costa Rica.

Looijen, JM. 1997. Environmental impact assessment: Lecture notes. International Institute for Aerospace Survey and Earth Sciences. 
Müller, S. 1996. ¿Cómo medir la sostenibilidad?: una propuesta para el área de la agricultura y de los recursos naturales. GTZ, San José, Costa Rica. (Serie Documentos de Discusión sobre Agricultura Sostenible y Recursos Naturales).

Murillo, L, L., Villalobos, F., Sáenz \& B., Vargas. 2004a. Un acercamiento integrado para determinar la sostenibilidad de granjas lecheras de Costa Rica: 1. Desarrollo de una matriz de indicadores. Revista electrónica Livestock Research for Rural Development 16 (Disponible en http:// www.cipav.org.co/Irrd/Irrd16/12/muri16096.htm.Consultado el 26 de ago 2011)

Murillo, L, L., Villalobos, F., Sáenz \& B., Vargas. 2004b. Un acercamiento integrado para determinar la sostenibilidad de granjas lecheras de Costa Rica: 2 . Cálculo de los índices de sostenibilidad. Revista electrónica Livestock Research for Rural Development 16 (Disponible en http://www.cipav.org.co/Irrd/Irrd16/12/muri16096.htm.Consultado el 26 de ago 2011)
Pezo, DA., L.A., Villegas \& F., Romero. 1993. Módulos lecheros ITCO/CATIE: Unaexperiencia de adaptación tecnológica a nivel de finca. In: Investigación con Pasturasen Fincas. Memorias VII Reunión del Comité Asesor de la Red Internacional deEvaluación de Pastos Tropicales (RIEPT), Palmira, Colombia, 27-29 de agosto de 1990. CIAT, Documento de Trabajo No. 124. CIAT, Cali, Colombia.

Ruiz, R. \& L.M., Oregui. 2001. El enfoque sistémico en el análisis de la producción animal: revisión bibliográfica. Investigación Agraria 16:29-61.

Villanueva, C., M., Ibrahim, K., Torres \& M., Torres. 2008. Planificación agroecológica de fincas ganaderas: la experiencia de la subcuenca Copán, Honduras. CATIE, Turrialba, Costa Rica.

Winograd, M. 1995. Indicadores ambientales para Latinoamérica y el Caribe: hacia la sustentabilidad en el uso de tierras. Proyecto IICA/GTZ. OEA, WRI. IICA, San José, Costa Rica. 